\section{Improved method for direct counting of basophil leucocytes}

\author{
J. R. COOPER AND C. N. D. CRUICKSHANK From \\ the Medical Research Council, Unit for Research \\ on the Experimental Pathology of the Skin, \\ Medical School, University, Birmingham
}

An improved method for the direct counting of basophil leucocytes from venous or capillary blood is described. The method is based upon the use of cetylpyridinium chloride to lyse the erythrocytes and render the mucopolysaccharides of the granule insoluble. Aluminium sulphate is used to mordant toluidine blue to the granules.

Our knowledge of the role of the basophil leucocytes in disease has been greatly hampered by the lack of a satisfactory method for their direct enumeration in capillary or venous blood. The essential difficulty lies in their relative paucity, so that in order to get sufficient numbers to count, concentrated blood solutions have to be used.

Most methods for counting basophils are based on the metachromatic staining of mucopolysaccharide in the basophil granules by aqueous solutions of toluidine blue. Saponin is used to lyse the erythrocytes and potassium or ammonium sulphate mixtures are sometimes added to keep the diluted blood in a fluid state (Boseila, 1959; Gurr, 1962).

In our hands these methods have been unsatisfactory from two points of view. The staining of the basophils is often diffuse because of the water solubility of the polysaccharides, making identification difficult and the count time consuming. Clotting and aggregation of platelets makes counting inaccurate and in addition the aggregation is often associated with the degranulation of the basophils in that area thus giving lower counts than the true value.

\section{MATERIALS AND METHOD}

The following method has been devised to overcome these difficulties.

Solutions Two solutions are required (1) $0 \cdot 1 \%$ E.D.T.A. in saline (disodium salt) and (2) $25 \mathrm{ml}$. of $0.5 \%$ cetylpyridinium chloride, $25 \mathrm{ml}$. of distilled water, $20 \mathrm{ml}$. of $0.8 \%$ toluidine blue in $5 \%$ aluminium sulphate, filtered before use.

Received for publication 15 November 1965.
METHOD Deliver to the bottom of a $75 \times 10 \mathrm{~mm}$. tub $0.08 \mathrm{ml}$. of solution 1 . Add $0.02 \mathrm{ml}$. of blood taken from free flowing thumb prick after wiping away the first drop: that exudes and mix by gentle shaking. Add $0.1 \mathrm{ml}$. of stain solution 2, mix and stopper. Fill two Fuchs=Rosenthal chambers using a Pasteur pipette, leave the chambers to stand in a moist chamber for five minutes to allow the cells to settle.

Basophils are seen as purple/red metachromaticall $\$$ stained cells. Other leucocytes, platelets, and erythrocytes are unstained. The use of E.D.T.A. as an anticoagulant eliminates the clotting of platelets. The cetylpyridiniunP chloride fulfils two functions: it lyses the erythrocytes and also complexes with mucopolysaccharides, rendering them relatively insoluble in water. The aluminiur sulphate accentuates the metachromatic staining of the basophils, by acting as a mordant (Heath, 1961).

\section{EVALUATION OF ERRORS}

Samples were taken from 10 normal healthy subjects iP order to assess the errors associated with pipetting and. counting. Three pipettes were used and two chamberswere counted from each pipette. The range of values obtained from these 10 subjects was 21 to 47 basophils per c.mm. of blood with a mean of 40 . A three-factos analysis of variance was carried out on the counts. Each of the averages for each subject was based on six results. It was found that the residual variance of the observation was 60.9 , which corresponded with a standard deviation of 7.8. Therefore, the standard deviation of each average was $\frac{7.8}{\sqrt{6}}$ or \pm 3.2 and the standard error of the differenc: between any two was $\frac{3 \cdot 2}{\sqrt{2}}$ or $2 \cdot 3$.

It is of interest that the error due to counting in the chambers was negligible and the major error was due t $\bar{\theta}$. pipetting. It is believed that the reduction of the totai error is due essentially to the improved staining techo nique. Such a small variance will render it possible to detect minor changes in basophil count with the minimur of labour. Counts may be completed in five to 10 minutes compared with some 30 to 60 minutes required by formen methods. Moreover, the dilution of blood is stable and as the staining is fast, counts may be done up to one week after making the mixture.

\section{REFERENCES}

Boseila, A. W. A. (1959). Stain Technol, 34, 335.

Gurr, E. (1962). Staining Animal Tissues, pp. 442-446. Leonard Hilu London.

Heath, I. D. (1961). Nature (Lond.), 191, 1370. 